\title{
Resveratrol ameliorates oxidative stress and inhibits aquaporin 4 expression following rat cerebral ischemia-reperfusion injury
}

\author{
WEINA LI ${ }^{1}$, CHANGHONG TAN ${ }^{2}$, YI LIU ${ }^{1}$, XI LIU $^{1}$, XIN WANG $^{1}$, YUEJIANG GUI ${ }^{1}$,

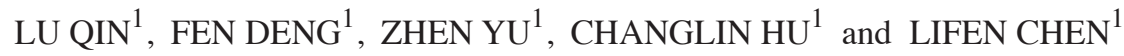 \\ ${ }^{1}$ Department of Neurology, The Second Affiliated Hospital of Chongqing Medical University, Chongqing 400010; \\ ${ }^{2}$ Department of Neurology, No. 6 Hospital of Chongqing, Chongqing 400060, P.R. China
}

Received May 23, 2014; Accepted August 14, 2015

DOI: $10.3892 / \mathrm{mmr} .2015 .4366$

\begin{abstract}
Cerebral ischemia-reperfusion (I/R) is associated with increased levels of reactive oxygen species (ROS) and brain edema, which lead to the deterioration of patient prognosis. Resveratrol serves a neuroprotective role in I/R injury, and this role may be associated with its anti-oxidative effects. However, resveratrol's mechanism of action in cerebral I/R injury remains to be fully understood. In order to investigate the effect of resveratrol in cerebral I/R-induced injury, male Sprague-Dawley rats were randomly assigned to four groups: The sham-operation group, the I/R group and the edaravone and resveratrol groups ( $\mathrm{I} / \mathrm{R}+\mathrm{E}$ and $\mathrm{I} / \mathrm{R}+\mathrm{R}$ groups). Infarct volume was evaluated by 2,3,5-tripenyltetrazolium chloride staining, brain edema was evaluated by the water content in the reperfused brain and malondialdehyde (MDA) was measured by the thiobarbituric acid method. Superoxide dismutase (SOD) levels were measured using the Total Superoxide Dismutase Assay kit. Inducible nitric oxide synthase (iNOS) levels in the hippocampus and cortex were measured by ELISA, and aquaporin 4 (AQP4) expression was measured by immunohistochemical staining and western blot analysis. The results demonstrated that resveratrol reduced the infarct volume and the incidence of brain edema and reduced neurological deficits. These outcomes were accompanied by reduced levels of MDA, iNOS and AQP4, and increased SOD levels in cerebral I/R injury. In conclusion, resveratrol protected against cerebral I/R injury by ameliorating oxidative stress and reducing AQP4 expression.
\end{abstract}

\footnotetext{
Correspondence to: Dr Lifen Chen, Department of Neurology, The Second Affiliated Hospital of Chongqing Medical University, 76 Linjiang Road, Chongqing 400010, P.R. China

E-mail: lifen_chen@163.com
}

Key words: resveratrol, stroke, cerebral ischemia-reperfusion, reactive oxygen species, inducible nitric oxide synthase, aquaporin 4

\section{Introduction}

Ischemic stroke results in long-term disability and is a leading cause of mortality in developed countries $(1,2)$. Early reperfusion is the most effective therapy for acute brain ischemia. However, reperfusion of the tissue is associated with increased production of reactive oxygen species (ROS), inflammation, necrosis and apoptosis, which worsen the prognosis of the patients (3). Therefore, reducing cerebral ischemia-reperfusion (I/R) injury is a key challenge in the treatment of cerebral ischemia.

The central event during cerebral ischemia is the generation of ROS, which results in damage to lipids, proteins and DNA, leading to neuronal death $(3,4)$. In a variety of ROS-generating systems, inducible nitric oxide synthase (iNOS) serves a major role by promoting the production of activated oxygen under hypoxic conditions, then further disrupting the blood-brain barrier and leading to brain edema $(5,6)$.

Brain edema, traditionally classified as vasogenic and cytotoxic edema, serves a critical role in cerebral I/R injury $(1,2,7)$. Cytotoxic edema occurs due to increased water permeability of cell membranes, which is associated with the aquaporin family, a group of selective water transport membrane proteins $(8,9)$. Aquaporin 4 (AQP4) is the predominant water channel and is distributed with a high density in the perivascular area and the supple astrocyte end-feet $(8,9)$. AQP4 is associated with brain edema in the mammalian brain; inhibition of AQP4 by a novel AQP4 inhibitor, TGN-020, was demonstrated to significantly reduce brain edema associated with focal ischemia (10).

Resveratrol is a polyphenol identified in grapes, berries, peanuts, medicinal plants (such as Polygonum cuspidatum and Veratrum grandiflorum) and red wine (11). Resveratrol has been proposed as a neuroprotective agent in the pathophysiological process of $I / R$ injury, and this function may be associated with its anti-oxidative and anti-inflammatory effects $(12,13)$. However, the mechanism by which resveratrol confers neuroprotection in cerebral ischemia remains to be fully elucidated. Edaravone has been reported to possess protective effects against ischemic brain damage in animal and clinical experiments $(14,15)$ and has been recommended as a potentially beneficial neuroprotective agent for the treatment of acute ischemic stroke by the Edaravone Acute Infarction Study Group (16). Therefore, in the present study, edaravone 
was selected as a positive control for investigating the effects of resveratrol in cerebral I/R injury.

The aim of the current study was to determine whether resveratrol has protective effects against I/R-induced injury in rats, and to verify whether resveratrol improves the neurological status and incidence of brain edema through the inhibition of oxidative stress and AQP4 expression.

\section{Materials and methods}

Animals and drugs. Adult male Sprague-Dawley rats weighing 250-300 g were provided by the Experimental Animal Center of Chongqing Medical University (Chongqing, China) and were provided with food and water ad libitum. The animal experiments were conducted in accordance with the guidelines of the Institutional Animal Care and Use Committee of Chongqing Medical University. Food and water were withdrawn $24 \mathrm{~h}$ prior to surgery. Resveratrol was purchased from Hongda Biotechnology Co., Ltd. (Linxia, China) and dissolved in water to prepare a $20 \%$ (w/v) solution. A superoxide dismutase (SOD) detection kit and an iNOS ELISA kit were purchased from Uscn Life Sciences, Inc. (Wuhan, China). Polyclonal anti-AQP4 antibodies (cat. no., LS-C3805-50; dilution, 1:50) were obtained from LifeSpan BioSciences (Seattle, WA, USA) 1:50). Additional general reagents were obtained from Nanjing Jiancheng Bioengineering Institute (Nanjing, China).

Induction of cerebral I/R. Transient focal cerebral I/R was produced by right middle cerebral artery occlusion (MCAO) as described previously (17). In brief, the rats were anesthetized with $300 \mathrm{mg} / \mathrm{kg}$ chloral hydrate (ChengDu Kelong Chemical Co., Ltd., Chengdu, China). Under an operating microscope, the right common carotid artery (CCA), the right external carotid artery (ECA) and the right internal carotid artery (ICA) were isolated and a 6-0 silk suture (Johnson \& Johnson (Shanghai) Medical Equipment Co., Ltd., Shanghai, China). was tied at the proximal and distal ends of the right ECA. The right ECA was then occluded. A 4-0 nylon monofilament (Johnson \& Johnson (Shanghai) Medical Equipment Co., Ltd.). was introduced into the right ECA and pushed up the ICA until light resistance was felt. The filament was inserted approximately $18-20 \mathrm{~mm}$ from the carotid bifurcation to effectively block the middle cerebral artery (MCA). The diameter of the tip of the suture was $\sim 300 \mu \mathrm{m}$. Following $90 \mathrm{~min}$ of MCAO, the suture was removed to restore blood flow (reperfusion confirmed by laser-Doppler flowmeter; Periflux System 5010, Perimed AB, Järfälla, Sweden). Sham-operated rats were manipulated in the same way, however the MCA was not occluded. Relative cerebral blood flow (rCBF) was monitored using the laser-Doppler flowmeter with the use of a flexible probe over the skull. $\mathrm{rCBF}$ was measured prior to ischemia, during MCAO and during reperfusion. Animals that did not demonstrate a $\mathrm{CBF}$ reduction of a minimum of $70 \%$ and animals that perished following ischemia induction were excluded from the experimental group. Neurological deficits were assessed in each animal on a numerical scale of $0-4$ at the moment of reperfusion and $24 \mathrm{~h}$ following reperfusion (18). Rating criteria: 0, no detectable deficits; 1 , forelimb flexion and torso turning to the contralateral side when lifted by the tail; 2, same behavior as grade 1 and decreased resistance to a lateral push; 3, same behavior as grade 2 with unilateral circling; 4, no spontaneous walking and a depressed level of consciousness. Rats with a neurological deficit less than 2 or greater than 3 were excluded from the study.

Drug treatment. Male rats $(\mathrm{n}=60)$ were randomly assigned to four groups ( $n=10 /$ group): i) Sham-operation group, received a single intraperitoneal injection of $0.9 \% \mathrm{NaCl}$; ii) I/R group, received an intraperitoneal injection of $0.9 \% \mathrm{NaCl} 10 \mathrm{~min}$ prior to operation in addition to at 0 and $20 \mathrm{~h}$ following reperfusion; iii) edaravone group, received an intraperitoneal injection of $10 \mathrm{mg} / \mathrm{kg}$ edaravone (Jilin Province Huinan Changlong Biopharmacy Co., Ltd., Jilin, China) 10 min prior to operation in addition to at 0 and $20 \mathrm{~h}$ following reperfusion; vi) resveratrol group, received an intraperitoneal injection of $20 \mathrm{mg} / \mathrm{kg}$ resveratrol at $10 \mathrm{~min}$ prior to operation in addition to at 0 and $20 \mathrm{~h}$ following reperfusion. The remaining 20 rats were removed from the experiments.

Cerebral infarct volume and edema measurement. Animals were sacrificed $48 \mathrm{~h}$ following reperfusion and brains were rapidly removed and frozen at $-20^{\circ} \mathrm{C}$ for $20 \mathrm{~min}$. The brains ( $\mathrm{n}=8$ /group) were sliced into $2 \mathrm{~mm}$-thick coronal sections and immersed in 2\% 2,3,5-tripenyltetrazolium chloride (TTC; Beijing Solarbio Science \& Technology Co., Ltd., Beijing, China) at $37^{\circ} \mathrm{C}$ for $50 \mathrm{~min}$ for staining of damaged tissue. Infarct size was measured using an image analysis system (Image-Pro Plus software, version 6.0; Media Cybernetics, Inc., Rockville, MD, USA). For the measurement of brain edema, the water content of the brain was assessed by a wet-dry method. Briefly, the brain slices were dried at $110^{\circ} \mathrm{C}$ for $24 \mathrm{~h}$ and the tissue water content was calculated as the percentage of the wet tissue weight as follows:

$\%$ water $=[($ wet weight - dry weight $) \times 100] /$ wet weight

Histological examination. Following transcardiac perfusion with $200 \mathrm{ml} \mathrm{4 \%}$ formaldehyde in $0.1 \mathrm{M}$ phosphate-buffered saline (PBS; pH 7.4), the rat brains were removed, post-fixed in $4 \%$ formaldehyde overnight and processed for paraffin embedding and cutting. Serial coronal sections (4-6 $\mu \mathrm{m}$ thick) were selected and processed for hematoxylin and eosin staining (Shanghai Hualan Chemical Technology Co., Ltd., Shanghai, China).

Lipid peroxidation and SOD activity assay. Brain tissues of the right hemisphere were homogenized in cold $0.1 \mathrm{~mol} / 1$ sodium phosphate buffer ( $\mathrm{pH}$ 7.4; Wuhan Boster Biological Technology, Ltd., Wuhan, China) to make a $10 \%$ homogenate. The homogenate was centrifuged for $15 \mathrm{~min}$ at $3,000 \mathrm{x} \mathrm{g}$ and $4^{\circ} \mathrm{C}$, and the supernatant was used for bioassays. The absorbance of the supernatant was measured spectrophotometrically (Shanghai Flash Spectrum Biological Technology Co., Ltd., Shanghai China) at $532 \mathrm{~nm}$ at room temperature and the results are expressed as nanomoles of $\mathrm{MDA} / \mathrm{mg}$ protein.

The production of superoxide was assessed by measuring the SOD-inhibited reduction of a water soluble tetrazolium salt (WST-1; Wuhan USCN Business Co., Ltd., Wuhan, China) as described previously $(19,20)$. Briefly, xanthine-xanthine oxidase (Wuhan USCN Business Co., Ltd.) was used to generate a superoxide flux. The absorbance obtained from a 
nitroblue tetrazolium (NBT; Wuhan USCN Business Co., Ltd.) reduction to blue formazan by superoxide was determined at $560 \mathrm{~nm}$ spectrophotometrically at room temperature. SOD in the sample competes for superoxide, inhibiting the rate of the reaction of superoxide with NBT. The rate of NBT reduction in the absence of tissue was used as the reference rate $(0.020 \pm 0.005$ absorbance units $/ \mathrm{min})$. The standard assay substrate mixture contained in $0.2 \mathrm{ml} 1 \mathrm{~mol} / \mathrm{l}$ xanthine (Wuhan USCN Business Co., Ltd.), 0.1 mol/1 EDTA (Nanjing Jiancheng Bioengineering Institute), $5.6 \times 10^{-2} \mathrm{~mol} / 1 \mathrm{NBT}$ and $1 \mathrm{mg} / \mathrm{ml}$ bovine serum albumin in $0.1 \mathrm{~mol} / 1$ sodium phosphate (pH 7.8) (both from Nanjing Jiancheng Bioengineering Institute).

iNOS activity assay. iNOS levels were measured with an iNOS ELISA kit according to the manufacturer's instructions. Briefly, the standards and test samples were added to pre-prepared rat iNOS monoclonal antibody ELISA plates, incubated with the plates for $2 \mathrm{~h}$ at $37^{\circ} \mathrm{C}$ and washed with scrubbing solution to remove unbound components. Enzyme working solution was then added, incubated for a sufficient amount of time, and washed with the wash buffer to remove unbound components. Solutions A, B and the 3,3',5,5'-tetramethylbenzidine (TMB) substrate were then sequentially added, followed by the stop solution which catalyzed the conversion of the blue substrate into a yellow product. iNOS levels were calculated from optical density measurements taken at a wavelength of $450 \mathrm{~nm}$, according to the values for the standards using Image-Pro Plus 6.0 (Media Cybernetics, Inc., Rockville, MD, USA)

AQP4 immunostaining. Brain tissues were removed following perfusion with $4 \%$ formaldehyde for tissue fixation, were embedded in paraffin and then serially sectioned $(4 \mu \mathrm{m})$. The rat brain sections were washed in $0.1 \mathrm{~mol} / \mathrm{l}$ PBS 10 times and placed in $3 \% \mathrm{H}_{2} \mathrm{O}_{2}$ to quench endogenous peroxidase activity for $15 \mathrm{~min}$. The sections were pre-incubated with $10 \%$ normal goat serum (Nanjing Jiancheng Bioengineering Institute) for $30 \mathrm{~min}$ at $37^{\circ} \mathrm{C}$ to block non-specific binding and incubated overnight at $4^{\circ} \mathrm{C}$ with the rabbit AQP4 antibody. Subsequently, the sections were washed in PBS and incubated with biotinylated anti-rabbit IgG antibodies (cat. no SA1028; Wuhan Boster Biological Technology, Ltd.) for $1 \mathrm{~h}$ at room temperature. Following washing, the sections were incubated for $30 \mathrm{~min}$ with streptavidin-biotin and horseradish peroxidase (Wuhan Boster Biological Technology, Ltd.) and developed with 3,3'-diaminobenzidine (Beijing Solarbio Science \& Technology Co., Ltd.). For negative controls, the primary antibodies were replaced with PBS. AQP4 expression was measured by the mean optical density (MOD) using Image-Pro Plus software, version 6.0.

Western blot analysis. Western blot analysis was used to measure AQP4 protein expression $48 \mathrm{~h}$ following I/R injury. Following transfer, the membrane was blocked in 5\% non-fat milk (Beijing Solarbio Science \& Technology Co., Ltd.) in TBST [1X Tris-HCL $(1,000 \mathrm{ml})$, Tween $20(1 \mathrm{ml})$ ] for $1 \mathrm{~h}$ at room temperature. The blots were incubated with rabbit polyclonal anti-AQP4 $(1: 2,000)$, or rabbit monoclonal anti- $\beta$-actin (1:2,000; sc-47778; Santa Cruz Biotechnology, Inc., Dallas, TX, USA) in 5\% non-fat milk and TBST overnight at $4^{\circ} \mathrm{C}$. Following three washes with $1 \mathrm{X}$ TBST for $5 \mathrm{~min}$ each, the blot was incubated with the horseradish peroxidase-conjugated goat anti-rabbit IgG antibody (1:4,000; cat. no. GAR007) MultiSciences, Lianke, Biotech Co., Ltd., Hanzhou, China) in TBST for $1 \mathrm{~h}$ at room temperature. Following washing with TBST, immunoreactive proteins were revealed with the enhanced chemiluminescence SuperSignal West Pico kit (Bio-Rad Laboratories, Inc., Hercules, CA, USA) on X-ray film (Bio-Rad Laboratories, Inc.), and the expression level was measured by densitometry. Band density values were normalized to that of $\beta$-actin.

Statistical analysis. Data are expressed as the mean \pm standard deviation. Statistical analyses of data were performed using one-way analysis of variance followed by the Student-Newman-Keuls test. $\mathrm{P}<0.05$ was considered to indicate a statistically significant difference. All data analyses were conducted using SPSS software, version 13.0 (SPSS, Inc., Chicago, IL, USA).

\section{Results}

Resveratrol protects against cerebral I/R-induced injury. At $24 \mathrm{~h}$ following reperfusion, the total infarct size was assessed by TTC staining with volumetric analysis. As presented in Fig. 1, there were no observed lesions or non-viable tissue in the sham-operated brains. By contrast, brain sections from the $\mathrm{I} / \mathrm{R}$ group exhibited lesions visible as white patches in the areas supplied by the MCA, which were observed overlaying the cortex and penetrating into the subcortical tissue. Resveratrol treatment significantly reduced $(\mathrm{P}<0.05)$ the lesions to $14.40 \pm 2.94 \%$ in the cerebral cortex and subcortical brain tissue compared with the I/R group. In the edaravone group, lesion size was significantly reduced $(\mathrm{P}<0.01)$ to $13.49 \pm 1.26 \%$ (Fig. 1F). Furthermore, cerebral I/R-induced injury significantly increased brain edema, which was inhibited by edaravone and resveratrol $(\mathrm{P}<0.01$; Fig. $1 \mathrm{G})$. Neurological deficits were measured $22 \mathrm{~h}$ following reperfusion. An increase in the severity of the neurological deficits was observed in the I/R group, however these were improved by $20 \mathrm{mg} / \mathrm{kg}$ resveratrol $(\mathrm{P}<0.01$ vs. the I/R group; Fig. 1E).

Resveratrol protects against cerebral I/R-induced histopathological alterations in the hippocampal CA1 region. As presented in Fig. 2, neurons of the hippocampal CA1 region in the sham-operated group were tightly packed with clear boundaries and no apoptosis or vacuolar degeneration was observed (Fig. 2A). Following cerebral I/R, the number of neurons was reduced and cell morphology became disrupted, with evidence of necrosis (Fig. 2B). In the I/R + R and I/R + E groups, the number of neurons was not observed to reduce, intact cell morphology was clearly visible and only a small fraction of cells exhibited cell death or vacuolar degeneration (Fig. 2C and D).

Resveratrol protects against cerebral I/R-induced oxidative stress. Chemocolorimetry was used to measure the brain MDA levels. As presented in Fig. 3A, cerebral I/R increased the brain MDA levels to $11.49 \pm 4.21 \mathrm{mg} / \mathrm{ml}$, whereas edaravone and resveratrol significantly reduced this level to $7.15 \pm 2.95$ and $6.53 \pm 1.94 \mathrm{mg}$ protein $/ \mathrm{ml}$, respectively $(\mathrm{P}<0.01$; 

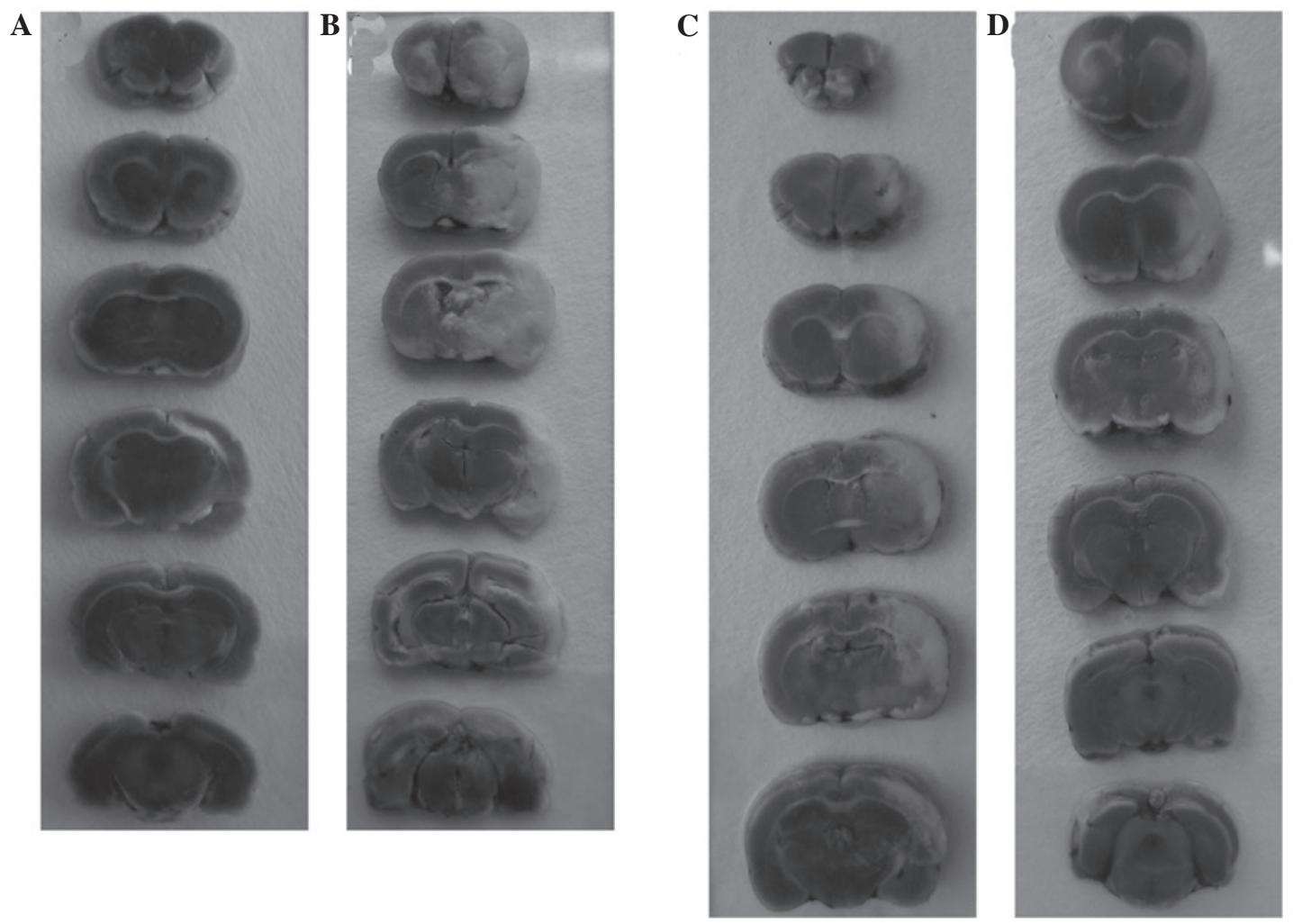

$\mathbf{E}$

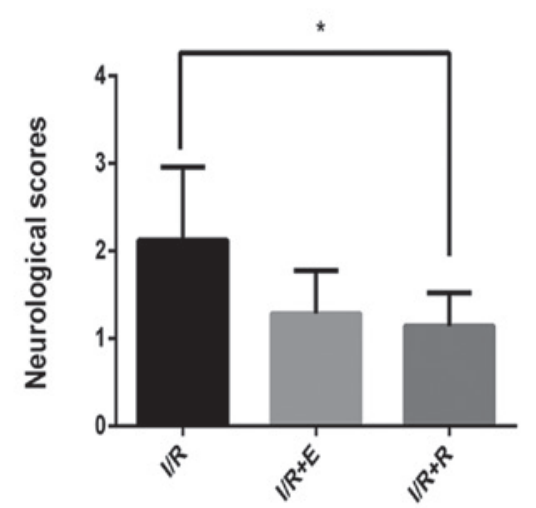

$\mathbf{F}$

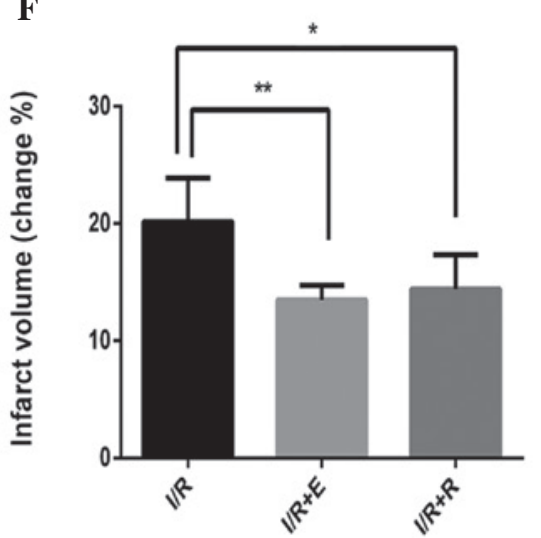

G

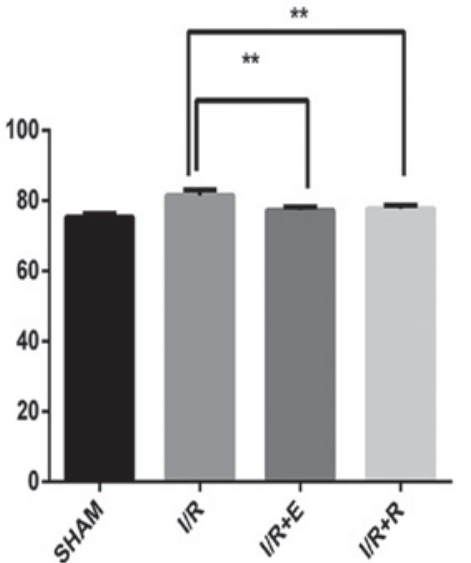

Figure 1.Resveratrol protected against cerebral I/R-induced injury in rats.(A-D) Representative images of brain sections stained with 2\% 2,3,4-tripenyltetrazolium chloride from (A) the sham-operated group, (B) the I/R group, (C) the I/R + E group and (D) the I/R + R group. (E) Measurement of neurological deficits following $22 \mathrm{~h}$ of reperfusion. (F) Measurement of infarct volume (vs. the I/R group) following $24 \mathrm{~h}$ of reperfusion. Percentage change was calculated as: Infarct volume/total volume x100. (G) Measurement of edema (vs. sham-operated brains) following 48 h of reperfusion. Percentage change was calculated as: (Wet weight - dry weight)/wet weight $\mathrm{x} 100 .{ }^{*} \mathrm{P}<0.05,{ }^{* *} \mathrm{P}<0.01$ vs. the I/R group. I/R, ischemia-reperfusion; E, edaravone; R, resveratrol.

Fig. 3A). Furthermore, cerebral I/R increased the iNOS levels in the focal ischemic cerebral cortex and hippocampus to $28.48 \pm 7.05 \mathrm{U} / \mathrm{mg}$ protein, compared with the sham control group (19.01 $\pm 5.56 \mathrm{U} / \mathrm{mg}$ protein). Resveratrol and edaravone significantly reduced iNOS levels to $20.17 \pm 6.49$ and $19.05 \pm 7.36 \mathrm{ng} / \mathrm{ml}$ respectively (Fig. 3B). Total SOD activity was measured in the cortex and hippocampus. As presented in Fig. 3C, SOD activity was increased with $24 \mathrm{~h}$ of reperfusion following MCAO. Furthermore, at $48 \mathrm{~h}$ of reperfusion following MCAO, the SOD activity level in the I/R group reduced to a lower level compared with the sham-operated group. Consistently, edaravone and resveratrol were highly effective at increasing SOD activity at 24 and $48 \mathrm{~h}$ of reperfusion following MCAO (Fig. 3C and D).
Resveratrol reduced cerebral I/R-induced AQP4 expression. At $48 \mathrm{~h}$ of reperfusion following MCAO, immunostaining for AQP4 expression was conducted in the cerebral hemispheres and the hippocampus in the four groups. As presented in Fig. 4, in the sham-operated group few AQP4-positive regions were observed (Fig. 4A). In the I/R group, the distribution of AQP4 immunostaining indicated diffuse, positive expression (Fig. 4B). In the edaravone and resveratrol treatment groups, reduced AQP4 expression was observed in astrocytes compared with the I/R group (Fig. 4C and D). To semi-quantify AQP4 expression, the MOD and the integrated optical density over the measurement area was calculated using Image-Pro Plus software, version 6.0 (Fig. 4E). Western blotting indicated 
A

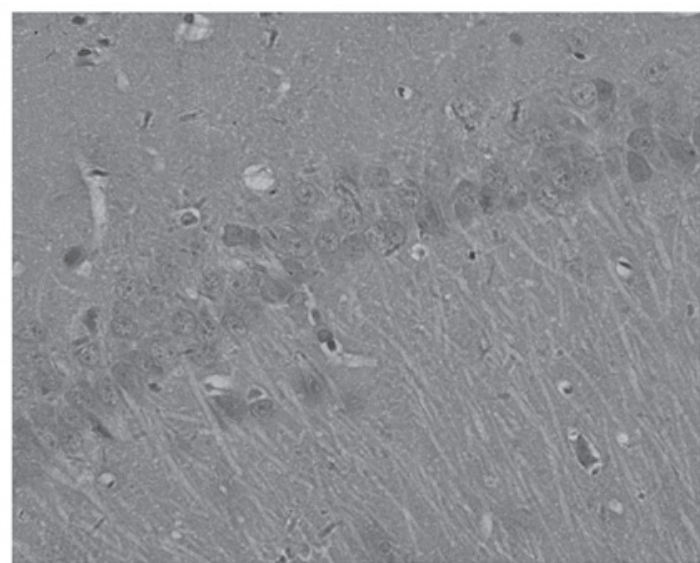

C

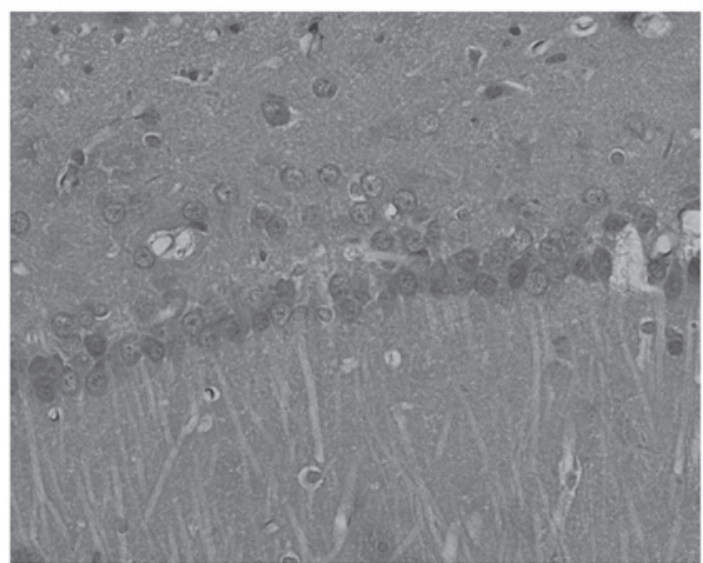

B

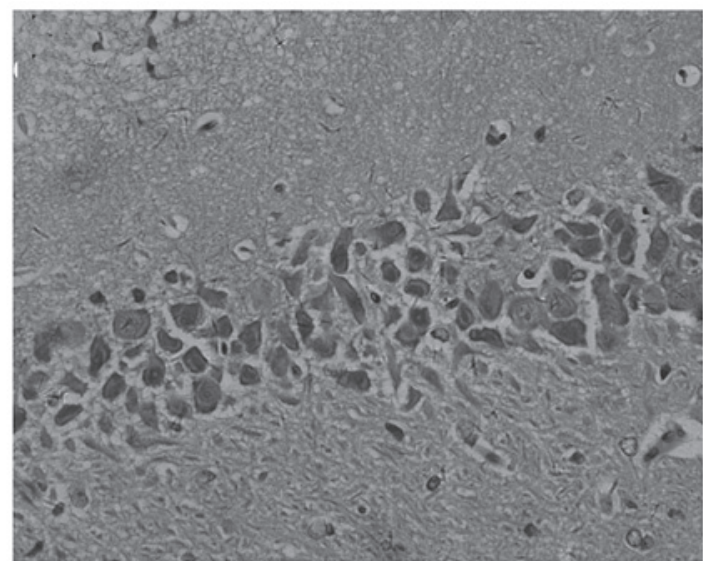

D

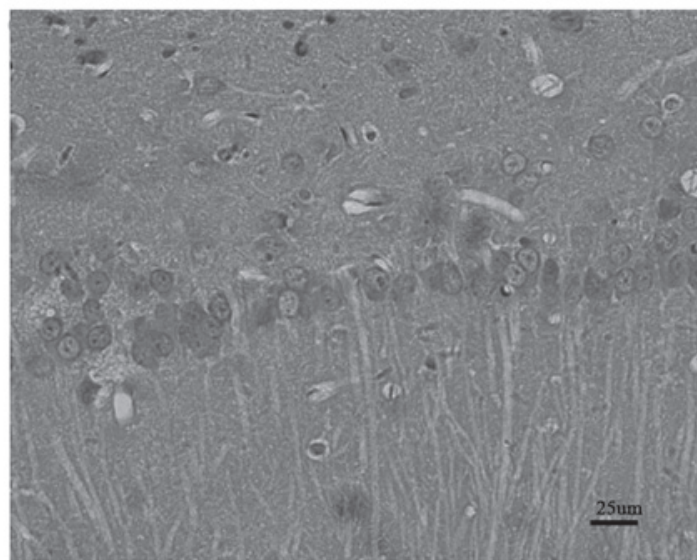

Figure 2. Resveratrol protected against cerebral I/R-induced histopathological alterations in the hippocampal CA1 region. Representative photographs of the hippocampal CA1 region stained by hematoxylin and eosin in (A) the sham-operated group, (B) the I/R group, (C) the I/R + edaravone group and (D) the $\mathrm{I} / \mathrm{R}+$ resveratrol group. Scale bar $=25 \mu \mathrm{m}$. I/R, ischemia-reperfusion.

A

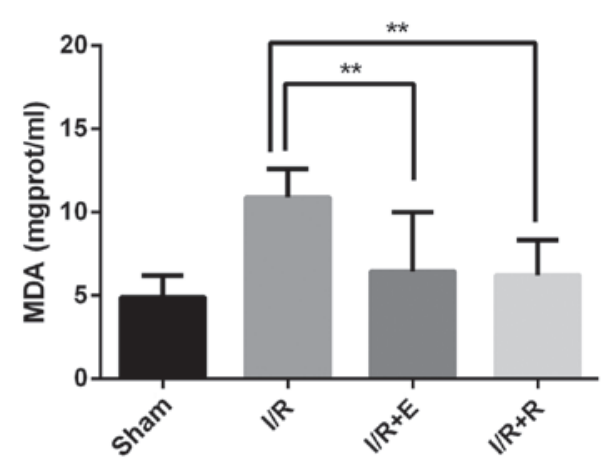

$\mathrm{C}$

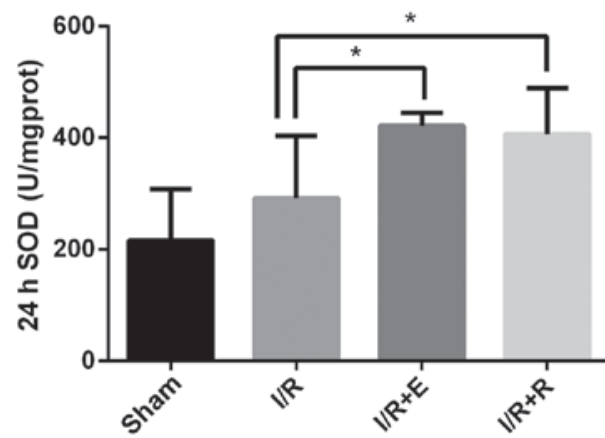

B

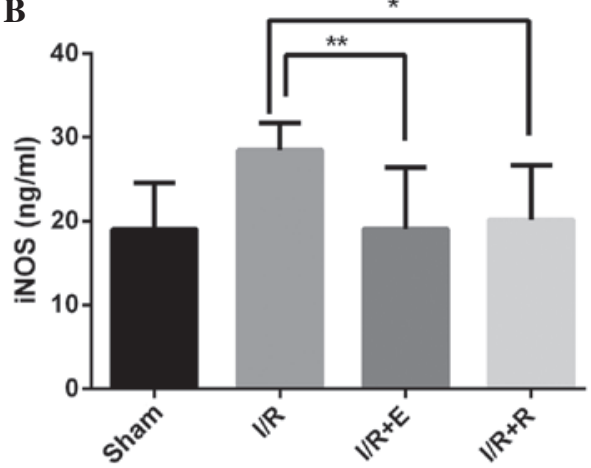

D

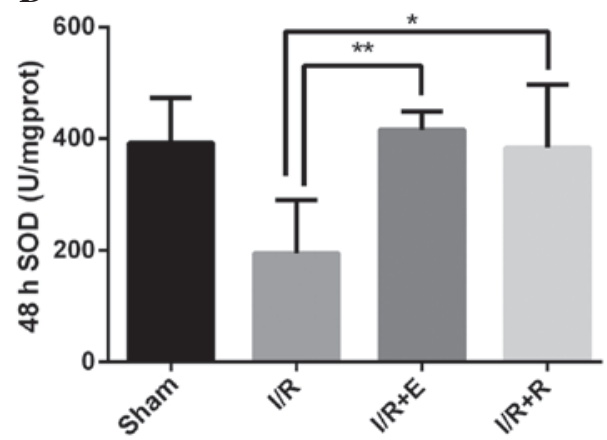

Figure 3. Resveratrol protected against cerebral I/R-induced oxidative stress. (A) MDA levels were measured following 90 min MCAO and 24 h reperfusion. (B) iNOS levels were measured following $90 \mathrm{~min}$ MCAO and $24 \mathrm{~h}$ reperfusion. SOD levels were measured at (C) $24 \mathrm{~h}$ and (D) $48 \mathrm{~h}$ of reperfusion following 90 min MCAO in rats. ${ }^{*} \mathrm{P}<0.05,{ }^{* *} \mathrm{P}<0.01$ vs. the $\mathrm{I} / \mathrm{R}$ group. ( $\mathrm{n}=8$ each group). I/R, ischemia-reperfusion; MDA, malondialdehyde; MCAO, middle cerebral artery occulsion; iNOS, inducible nitric oxide synthase; SOD, superoxide dismutase; E, edaravone; R, resveratrol. 


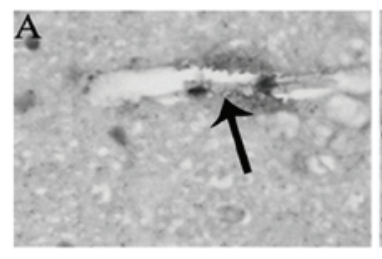

$\mathbf{E}$
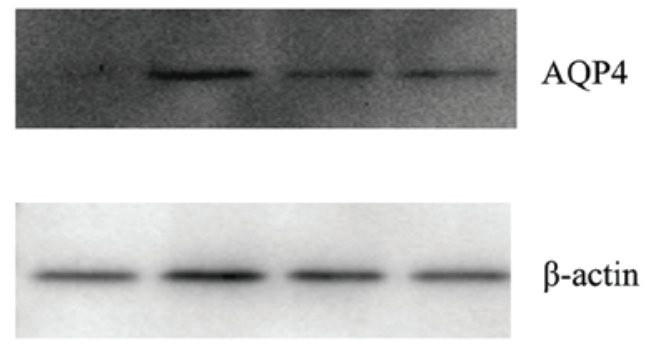
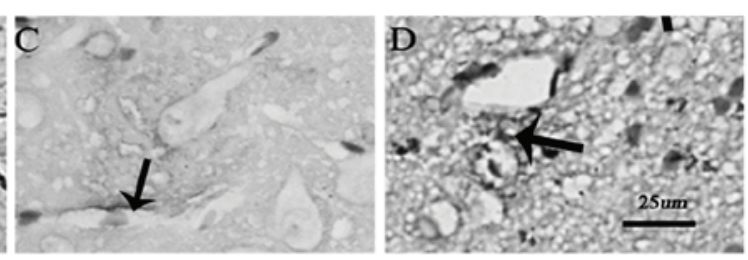

F

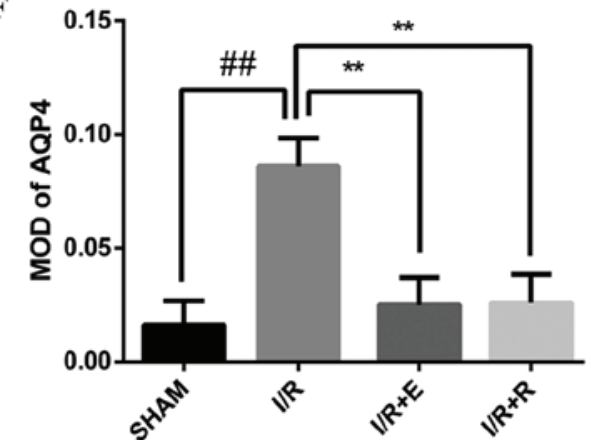

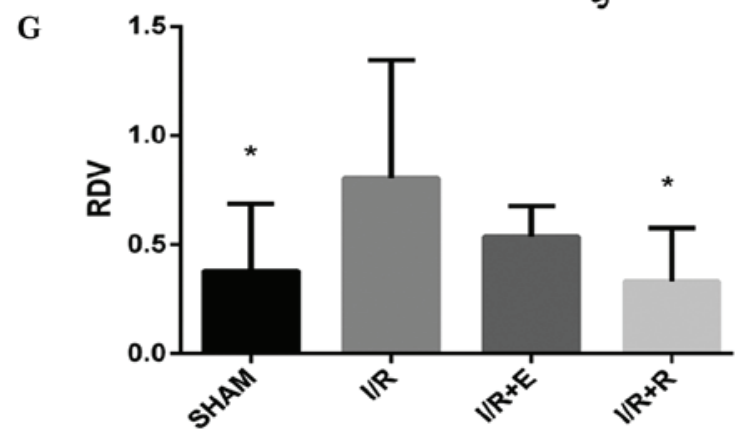

Figure 4. Resveratrol reduced cerebral I/R-induced AQP4 expression. Representative AQP4 staining of the hippocampus in (A) the sham-operated group, (B) the I/R group, (C) the $I / R+E$ group and (D) the I/R $+R$ group. Scale bar $=25 \mu \mathrm{m}$. (E) Western blot of AQP4 immunoreactivity. From left to right in sequence: Sham group, I/R group, I/R+E group,I/R+R group (F) AQP4 expression $48 \mathrm{~h}$ following cerebral I/R. (G) Relative density of AQP-4 to $\beta$-actin protein expression following cerebral I/R. ${ }^{P} \mathrm{P}<0.05,{ }^{* *} \mathrm{P}<0.01$ vs. the I/R group; ${ }^{\prime} \mathrm{P}<0.05,{ }^{\# *} \mathrm{P}<0.01$ vs. the sham-operated group ( $\mathrm{n}=8$ each group). I/R, ischemia-reperfusion; AQP4, aquaporin 4, E, edaravone; R, resveratrol; MOD, mean optical density; RD, relative density.

that AQP4 expression was significantly increased following cerebral I/R compared with the control group, however this was inhibited by pre-treatment with edaravone and resveratrol (Fig. 4F and G). Both edaravone and resveratrol significantly reduced the infarct size; however, in the aspect of reducing neurological deficits, no statistically significant differences were detected between the edaravone and control treatment groups.

\section{Discussion}

In the current study, it was demonstrated that treatment with resveratrol significantly reduced infarct volume and brain edema following cerebral I/R injury, in addition to reducing the levels of MDA, iNOS, AQP4 and apoptosis, improving the neurological deficit score and increasing SOD levels. Thus, resveratrol may protect against cerebral I/R injury by ameliorating oxidative stress and reducing $\mathrm{AQP} 4$ expression.

Numerous mechanisms have been implicated in the pathology of cerebral I/R damage, including excitotoxicity, oxidative stress, inflammation and apoptosis (21). I/R injury leads to the generation of ROS, including superoxide $\left(\mathrm{O}_{2}^{-}\right)$, hydrogen peroxide $\left(\mathrm{H}_{2} \mathrm{O}_{2}\right)$, hydroxyl radicals $(\mathrm{OH} \bullet)$ and peroxynitrite $\left(\mathrm{ONOO}^{-}\right)$(22). ROS trigger lipid peroxidation and result in cell damage by increasing the permeability of the cell membrane, promoting MDA generation and DNA damage (23). Exposure to ischemia and hypoxia generates inflammatory cytokines, which induce excess NO generation and iNOS synthesis, and in turn stimulate the production of ROS. For example, $\bullet N O$ reacts with $\mathrm{O}_{2} \bullet$ forming $\mathrm{ONOO}^{-}$which forms its acid form, peroxynitrous acid $(\mathrm{ONOOH})$, an unstable and reactive oxidizing species (22). It has been demonstrated that iNOS produces NO with neurotoxic effects (24). In the current study, $24 \mathrm{~h}$ of reperfusion following $90 \mathrm{~min} \mathrm{MCAO}$ was observed to increase the levels of MDA and iNOS in the brain, with this effect inhibited by treatment with resveratrol. Furthermore, resveratrol inhibited iNOS production, reduced infarct volume and neurological deficits in addition to reducing neuronal apoptosis following cerebral ischemia. Therefore, the resveratrol-mediated protective effects in cerebral I/R injury may be achieved by reducing the level of MDA, improving SOD activity and suppressing rising iNOS levels.

Brain edema in ischemic stroke is known to occur via two mechanisms, cytotoxic edema and vasogenic edema. Cytotoxic edema is associated with an impairment of cellular metabolism resulting in the swelling of astrocytes, neurons and dendrites, whilst vasogenic edema is followed by leakage of the blood-brain barrier (25). Astrocytic swelling through AQP4 in the plasma membrane serves an important role in cytotoxic edema (26). Knockout of AQP4 or disruption of its 
polarized expression pattern mitigated the brain water accumulation associated with brain ischemia, water intoxication and hyponatremia (26). It was reported that resveratrol serves a role in mitigating brain edema $(21,27)$. In the current study, resveratrol was demonstrated to inhibit I/R-induced cerebral edema associated with reduced AQP4 expression. These data demonstrate that resveratrol has potential in conferring neuroprotection, by reducing free radical damage via the suppression of ROS generation, and the upregulation of the endogenous defense system against free radicals. In addition, resveratrol may further reduce brain edema by inhibiting AQP4 expression. In conclusion, the results presented in the current study may contribute to the future translational application of resveratrol in patients with ischemic stroke.

\section{Acknowledgements}

The current study was supported by the Natural Science Foundation Project of CQ CSTC (grant no. 2011BB5140). The funding bodies had no role in study design, data collection and analysis, decision to publish or manuscript preparation. The authors would like to thank Chongqing Key Laboratory of Neurology, Chongqing Medical University for their help in the current study.

\section{References}

1. Moskowitz MA, Lo EH and Iadecola C: The science of stroke: Mechanisms in search of treatments. Neuron 67: 181-198, 2010.

2. Fan X, Qiu J, Yu Z, Dai H, Singhal AB, Lo EH and Wang X: A rat model of studying tissue-type plasminogen activator thrombolysis in ischemic stroke with diabetes. Stroke 43: 567-570, 2012.

3. Olmez I and Ozyurt H: Reactive oxygen species and ischemic cerebrovascular disease. Neurochem Int 60: 208-212, 2012.

4. Wilkinson-Berka JL, Rana I, Armani R and Agrotis A: Reactive oxygen species, Nox and angiotensin II in angiogenesis: Implications for retinopathy. Clin Sci (Lond) 124: 597-615, 2013

5. Medeiros A, Peres-Buzalaf C, Fortino Verdan F and Serezani CH: Prostaglandin E2 and the suppression of phagocyte innate immune responses in different organs. Mediators Inflamm 2012: 327568, 2012.

6. Dai H, Yu Z, Fan X, Liu N, Yan M, Chen Z, Lo EH, Hajjar KA and Wang X: Dysfunction of annexin A2 contributes to hyperglycaemia-induced loss of human endothelial cell surface fibrinolytic activity. Thromb Haemost 109: 1070-1078, 2013.

7. Li W, Liu J, He P, Ni Z, Hu Y, Xu H and Dai H: Hydroxysafflor yellow A protects methylglyoxal-induced injury in the cultured human brain microvascular endothelial cells. Neurosci Lett 549: 146-150, 2013.

8. Gunnarson E, Zelenina M and Aperia A: Regulation of brain aquaporins. Neuroscience 129: 947-955, 2004.

9. Bonomini F and Rezzani R: Aquaporin and blood brain barrier. Curr Neuropharmacol 8: 92-96, 2010.
10. Igarashi H, Huber VJ, Tsujita M and Nakada T: Pretreatment with a novel aquaporin 4 inhibitor, TGN-020, significantly reduces ischemic cerebral edema. Neurol Sci 32: 113-116, 2011.

11. Hao HD and He LR: Mechanisms of cardiovascular protection by resveratrol. J Med Food 7: 290-298, 2004.

12. Panickar KS, Polansky MM and Anderson RA: Cinnamon polyphenols attenuate cell swelling and mitochondrial dysfunction following oxygen-glucose deprivation in glial cells. Exp Neurol 216: 420-427, 2009.

13. Yousuf S, Atif F, Ahmad M, Hoda N, Ishrat T, Khan B and Islam F: Resveratrol exerts its neuroprotective effect by modulating mitochondrial dysfunctions and associated cell death during cerebral ischemia. Brain Res 1250: 242-253, 2009.

14. Ahmad A, Khan MM, Javed H, Raza SS, Ishrat T, Khan MB, Safhi MM and Islam F: Edaravone ameliorates oxidative stress associated cholinergic dysfunction and limits apoptotic response following focal cerebral ischemia in rat. Mol Cell Biochem 367: 215-225, 2012.

15. Nonaka Y, Shimazawa M, Yoshimura S, Iwama T and Hara H: Combination effects of normobaric hyperoxia and edaravone on focal cerebral ischemia-induced neuronal damage in mice. Neurosci Lett 441: 224-228, 2008.

16. Effect of a novel free radical scavenger, edaravone (MCI-186), on acute brain infarction. Randomized, placebo-controlled, double-blind study at multicenters. Cerebrovasc Dis 15: 222-229, 2003.

17. Longa EZ, Weinstein PR, Carlson S and Cummins R: Reversible middle cerebral artery occlusion without craniectomy in rats. Stroke 20: 84-91, 1989.

18. Bederson JB, Pitts LH, Tsuji M, Nishimura MC, Davis RL and Bartkowski H: Rat middle cerebral artery occlusion: Evaluation of the model and development of a neurologic examination. Stroke 17: 472-476, 1986.

19. Kravcukova P, Danielisova V, Nemethova M, Burda J and Gottlieb M: Transient forebrain ischemia impact on lymphocyte DNA damage, glutamic acid level, and SOD activity in blood. Cell Mol Neurobiol 29: 887-894, 2009.

20. Beauchamp C and Fridovich I: Superoxide dismutase: Improved assays and an assay applicable to acrylamide gels. Anal Biochem 44: 276-287, 1971.

21. Khan MM, Ahmad A, Ishrat T, Khuwaja G, Srivastawa P, Khan MB, Raza SS, Javed H, Vaibhav K, Khan A and Islam F: Rutin protects the neural damage induced by transient focal ischemia in rats. Brain Res 1292: 123-135, 2009.

22. Chan PH: Role of oxidants in ischemic brain damage. Stroke 27: 1124-1129, 1996.

23. Inoue M, Sato EF, Nishikawa M, Park AM, Kira Y, Imada I and Utsumi K: Mitochondrial generation of reactive oxygen species and its role in aerobic life. Curr Med Chem 10: 2495-2505, 2003.

24. Gilg AG, Singh AK and Singh I: Inducible nitric oxide synthase in the central nervous system of patients with X-adrenoleukodystrophy. J Neuropathol Exp Neurol 59: 1063-1069, 2000

25. Danielisová V, Némethová M, Gottlieb M and Burda J: Changes of endogenous antioxidant enzymes during ischemic tolerance acquisition. Neurochem Res 30: 559-565, 2005.

26. Fukuda AM and Badaut J: Aquaporin 4: A player in cerebral edema and neuroinflammation. J Neuroinflammation 9: 279, 2012.

27. Wang YJ, He F and Li XL: The neuroprotection of resveratrol in the experimental cerebral ischemia. Zhonghua Yi Xue Za Zhi 83: 534-536, 2003 (In Chinese). 